\title{
TTR
}

Traduction, terminologie, re?daction

\section{Jean Darbelnet l'homme, sa pensée et son action}

\section{Geneviève Mareschal}

Volume 4, numéro 1, 1st semester 1991

Languages and Cultures in Translation Theories

URI : https://id.erudit.org/iderudit/037087ar

DOI : https://doi.org/10.7202/037087ar

Aller au sommaire du numéro

Éditeur(s)

Association canadienne de traductologie

ISSN

0835-8443 (imprimé)

1708-2188 (numérique)

Découvrir la revue

Citer cet article

Mareschal, G. (1991). Jean Darbelnet l'homme, sa pensée et son action. TTR, 4(1), 145-150. https://doi.org/10.7202/037087ar d'utilisation que vous pouvez consulter en ligne.

https://apropos.erudit.org/fr/usagers/politique-dutilisation/ 


\title{
Jean Darbelnet l'homme, sa pensée et son action
}

\author{
Geneviève Mareschal
}

Très sensible à l'honneur qui m'est donné ici de retracer dans ses grandes lignes la carrière et la pensée de Jean Darbelnet, j'aimerais commencer par remercier la direction de TTR de l'hommage qu'il m'est ainsi permis de rendre à un homme remarquable, à un éminent comparatiste du français et de l'anglais et à un universitaire passionné par son métier. Je tiens à associer à cet hommage ses étudiants et disciples, ses collègues et amis, et surtout tous ceux qui, comme moi, ont eu la chance et le privilège de le connaître un peu mieux et un peu plus intimement.

Quand, en 1923, Jean Darbelnet se destine à l'enseignement de l'anglais et entreprend des études supérieures à la Sorbonne, il se fixe comme objectif «la possession aussi poussée que possible de l'anglais»: il voulait, disait-il, parler et écrire l'anglais «à peu près» comme un Anglais ou un Américain instruit.' Conscient de l'envergure de l'entreprise, il met à profit les séjours prolongés qu'il effectue pendant cinq ans en Angleterre comme lecteur de français, pour parfaire sa connaissance de l'anglais. Très vite, il s'aperçoit que la "possession poussée» d'une langue suppose non seulement la connaissance des mots, mais aussi de leur emploi idiomatique et du niveau de langue auquel ils se situent, toute erreur d'emploi ou de niveau trahissant l'étranger au même titre qu'une faute d'accentuation.

1. Propos tenus dans un texte rédigé en 1982, à la demande du président de la Société des écrivains canadiens. 
Cet apprentissage personnel d'une langue seconde à un niveau avancé va lui faire prendre pleinement conscience de l'importance de la «stylistique» dans l'expression langagière, et par «stylistique» il entend, dans l'optique du linguiste genevois Charles Bally, l'utilisation correcte et efficace des ressources de la langue en fonction des situations dans lesquelles elle est employée et de la culture du locuteur. Quand les circonstances de la vie vont, en terre d'Amérique, orienter sa carrière vers l'enseignement du français aux anglophones, l'importance de la stylistique dans la maîtrise d'une langue va s'imposer à lui avec plus d'acuité encore.

Cette double prise de conscience, l'une inspirée par sa propre expérience, l'autre par ses activités d'enseignant, est à l'origine du développement de sa pensée linguistique, toute entière fondée sur la réalité de la langue en situation. Et l'observation du français au Canada en contexte bilingue viendra par ailleurs confirmer les fondements de sa pensée.

Cette pensée linguistique, Jean Darbelnet l'énonce dans la Stylistique comparée du français et de l'anglais, qu'il publie en 1958 , en collaboration avec Jean-Paul Vinay. Cet ouvrage, qui constitue sans contredit une étape et une publication majeures dans la carrière de Jean Darbelnet, jette les bases d'une réflexion théorique et pratique sur les différences et écarts caractéristiques qui existent entre le français et l'anglais, en mettant en évidence les moyens d'expression et les procédés stylistiques qui permettent de produire les mêmes effets dans les deux langues, sans pour autant faire appel aux mêmes éléments linguistiques. Ce manuel et les exercices qui l'accompagnent reçoivent un accueil très favorable dans les milieux linguistiques. Bien des spécialistes de l'anglais et du français s'y reconnaissent, et les traducteurs y trouvent un «livre à penser». Dans les dédales que constitue le passage d'une langue à l'autre, la Stylistique comparée offre pour la première fois «un effort d'organisation et de classement des difficultés et pièges qui attendent les traducteurs dans le domaine français/anglais, le tout basé sur un grand nombre d'exemples»'. Il faut y voir, non pas, comme certains ont voulu l'avancer, une théorie de la traduction, mais l'énoncé d'un certain nombre de principes et de mécanismes linguistiques visant à préserver et à garantir le caractère spécifique et idiomatique des langues en présence. Les auteurs y ont certaine-

2. Jean-Paul Vinay, «SCFA Revisited», Meta (vol. 28, n 4, 1983, p. 419). 
ment fait cuvre de pionniers et le nombre de travaux qui s'inspirent de leur pensée linguistique démontrent à l'évidence l'importance et la fécondité de celle-ci.

Ce travail de réflexion sur le français et l'anglais, Jean Darbelnet l'a poursuivi toute sa vie, traquant avec méthode et objectivité, les écarts séparant les deux langues. La «linguistique différentielle», comme il avait choisi et pris l'habitude de qualifier sa spécialité, et qu'il définissait comme «l'analyse des analogies et des différences entre deux langues aussi bien au niveau des structures qu'à celui des significations et des valeurs $»^{3}$, se fondait sur le fait que «les langues ne découpent pas la réalité de la même façon, qu'elles ne mettent pas les mêmes structures au service des mêmes significations et qu'elles donnent souvent un sens différent à des structures parallèles ou identiques»".

Quand Jean Darbelnet sera nommé professeur à l'Université Laval en 1962, ses recherches et ses écrits dans le domaine de la linguistique différentielle lui valent déjà une renommée qui dépasse de beaucoup les frontières du Québec et du Canada. Menant de front ses activités d'enseignement et ses travaux de recherche, et alimentant les premières avec les résultats des seconds, il pousse plus loin l'analyse de sa discipline en affinant ses méthodes de collecte des faits langagiers, leur classement et leur interprétation. Même si, par la qualité des analyses qu'il propose, Jean Darbelnet se situe aux confins de la réflexion théorique, il aimait revendiquer le titre bien modeste de «data collector", et tous ceux qui l'ont connu se souviendront de l'avoir vu noter sur un petit carnet, qu'il avait toujours sur lui, tel mot qu'il venait de lire ou entendre, telle tournure de phrase qui l'avait frappé, telle construction qui méritait d'être vérifiée.

Bien que le Québec ne soit pas une terre inconnue pour Jean Darbelnet, puisqu'il avait enseigné et occupé les fonctions de directeur au département d'études françaises de l'Université McGill de 1940 à 1946, c'est à partir de 1957 qu'il a véritablement renoué avec cette province et les préoccupations linguistiques de celle-ci. En fait, les retrouvailles de Jean Darbelnet avec le Québec ouvrent

3. Jean Darbelnet, «Linguistique différentielle et traduction», Meta (vol. 16, nं 1-2, 1971, p. 18).

4. Ibid., p. 17. 
pour lui une phase nouvelle et très enrichissante de sa carrière et de son activité intellectuelle, une phase où le français va occuper une place de plus en plus prédominante.

Invité à tenir une chronique bimensuelle de français intitulée «La langue et la vie» dans l'hebdomadaire montréalais Notre temps, Jean Darbelnet va rédiger, entre 1957 et 1962, plus d'une centaine d'articles sur l'usage du français au Québec et en France. La moitié de ces articles ont été reproduits dans Regards sur le français actuel, livre paru chez Beauchemin en 1963 et aujourd'hui épuisé. C'est cette chronique qui va éveiller chez Jean Darbelnet sa vocation de «défenseur du français», vocation qui va se catalyser autour de la lutte contre l'anglicisation. Au Québec, Jean Darbelnet se trouve en effet au cœur du combat, dans un pays où le français est en contact direct et quotidien avec l'anglais et où cette langue se trouve continuellement menacée dans son existence et son intégrité par la présence envahissante de l'anglais. Jean Darbelnet ne pouvait rêver mieux! Après avoir réfléchi pendant des années aux différences qui séparent le français de l'anglais, il était admirablement préparé pour dénoncer le danger et y faire face. Jean Darbelnet trouve dans le débat sur le français au Québec un champ d'étude et d'intervention particulièrement riche. Sans tomber dans le militantisme, il va s'employer à dénoncer l'influence perturbatrice et souvent dévastatrice que l'anglais a sur le français au Québec et au Canada. Inlassablement, il va relever les anglicismes qui envahissent le vocabulaire français et les structures du français. Inlassablement aussi, il va proposer des termes de remplacement et des solutions de rechange. Il est aux avant-postes de tous les efforts de correction de la langue et de francisation entrepris par les instances politiques québécoises et canadiennes et par les institutions d'enseignement. Sa compétence en la matière et la qualité de son jugement en font un consultant recherché et écouté.

Le rapport qu'il rédige en 1965 pour le compte de la Commission royale d'enquête sur le bilinguisme et le biculturalisme porte expressément sur «l'anglicisation de la langue française au Québec, ses causes et ses remèdes possibles» et la typologie des anglicismes qu'il y propose sera par la suite reproduite dans un livre publié en 1976 sous le titre le Français en contact avec l'anglais en Amérique du Nord. Ce titre sera partiellement repris par Maurice Pergnier en 1988 lorsqu'il offrira en hommage à Jean 
Darbelnet un collectif entièrement inspiré du combat mené par celui-ci contre l'anglicisation du français.'

Ce bref survol de la pensée et de l'action de Jean Darbelnet est loin d'être complet, et il ne le serait sûrement pas si l'on omettait de mentionner ici son apport à la «traduction». Sans oublier le rôle de premier plan que Jean Darbelnet a joué dans la mise sur pied d'ateliers, de cours et de programmes de traduction tant au niveau universitaire que dans le cadre d'associations professionnelles de traducteurs ou autres spécialistes de la langue, c'est sa conception de la traduction que j'aimerais mettre en relief ici. Que ce soit dans son enseignement ou dans les nombreux articles qu'il a consacrés au sujet, Jean Darbelnet a toujours mis en garde ses contemporains contre la «tyrannie de la forme». Il ne fait aucun doute dans son esprit que la traduction doit se placer au niveau du sens et de la transmission du sens si l'on veut éviter, comme il se doit, le piège du littéralisme et les aléas de la mauvaise traduction. La traduction, où il voit un champ d'application privilégié pour la linguistique différentielle, requiert selon lui une formation méthodique. «Le temps n'est plus, disait-il, où l'on pouvait s'improviser traducteur sous prétexte qu'on savait deux langues. Les traducteurs de génie, qui n'ont pas besoin d'être formés, sont trop peu nombreux pour suffire à nos besoins croissants. ${ }^{6}$ Cette formation passe par l'obligation de faire bien comprendre aux futurs traducteurs que, dans le passage d'une langue à l'autre, le sens et la forme ne sont pas forcément liés et que, pour faire une bonne traduction, il faut traduire des idées et non des mots. Le découpage en unités de traduction, la recherche d'équivalences sémantiques et stylistiques, le respect de la tonalité de l'original, voilà quelquesuns des principes traductionnels qu'il a essayé d'illustrer, de défendre et d'inculquer.

Enfin, il n'est pas possible de rendre à Jean Darbelnet tout l'hommage qu'il mérite sans évoquer l'homme qu'il était. Honnê-

5. Le Français en contact avec l'anglais. En hommage à Jean Darbelnet. Travaux réunis par Maurice Pergnier (Paris, Didier Érudition, 1988). On trouve aussi dans ce collectif une bibliographie presque complète des ouvrages et articles de Jean Darbelnet, aux pages 57-63.

6. Jean Darbelnet, «Linguistique différentielle et traduction», loc. cit., p. 17. 
teté et intégrité sont sans nul doute les mots qui viennent automatiquement à l'esprit de quiconque l'a connu et fréquenté. Tant sur le plan intellectuel que moral, Jean Darbelnet était un homme de principes, et, au nom de ceux-ci, il était prêt à mener les luttes qui s'imposaient. Il n'était certes pas homme de compromis et, lorsqu'il était amené à en faire, c'était à son corps défendant, généralement parce que ceux-ci lui étaient imposés. Exigeant envers lui-même, il l'était aussi envers les autres, mais ceux qui étaient parvenus à gagner sa confiance savaient qu'ils pouvaient compter sur lui et sur son appui indéfectible.

Sous des dehors parfois bourrus, Jean Darbelnet était un homme sociable et affable. Il aimait la compagnie, les discussions stimulantes et les soirées en petit comité autour d'une bonne table. Sa grande culture et son érudition en faisaient un interlocuteur apprécié. Et il aimait par-dessus tout confronter ses idées, ses réflexions et ses interrogations avec les gens qu'il respectait.

«Je suis de la vieille école», disait-il pour expliquer son attachement aux valeurs traditionnelles de la vie telles que la famille, la politesse, le respect d'autrui et celui de la parole donnée. Et son comportement reflétait en tous points cette philosophie de vie.

Travailleur infatigable, il avait toujours en chantier un article, une étude ou un ouvrage plus conséquent. Sa dernière publication importante, un petit Dictionnaire des particularités de l'usage paru en 1986 aux Presses de l'Université du Québec, têmoigne de cette ardeur au travail que l'âge n'avait en rien amoindrie.

Les hautes distinctions qu'il s'est vu décerner par les gouvernements français et canadien, et les titres de Membre de la Société royale du Canada et de Doctor honoris causa de l'Université d'Ottawa qu'il portait avec tant d'honneur et de fierté, n'ont été que le juste tribut versé à un homme, à sa pensée et à son action. 